\title{
QUEEN'S
UNIVERSITY
BELFAST
}

\section{Towards a spectroscopically accurate set of potentials for heavy hydride laser cooling candidates: effective core potential calculations of $\mathrm{BaH}$}

Moore, K., McLaughlin, B. M., \& Lane, I. C. (2016). Towards a spectroscopically accurate set of potentials for heavy hydride laser cooling candidates: effective core potential calculations of BaH. Journal of Chemical Physics.

Published in:

Journal of Chemical Physics

Document Version:

Peer reviewed version

Queen's University Belfast - Research Portal:

Link to publication record in Queen's University Belfast Research Portal

\author{
Publisher rights \\ (C) 2016 The Authors \\ The following article has been accepted by Journal of Chemical Physics. After it is published, it will be found at \\ http://scitation.aip.org/content/aip/journal/jcphttp://scitation.aip.org/content/aip/journal/jcp
}

\section{General rights}

Copyright for the publications made accessible via the Queen's University Belfast Research Portal is retained by the author(s) and / or other copyright owners and it is a condition of accessing these publications that users recognise and abide by the legal requirements associated with these rights.

\section{Take down policy}

The Research Portal is Queen's institutional repository that provides access to Queen's research output. Every effort has been made to ensure that content in the Research Portal does not infringe any person's rights, or applicable UK laws. If you discover content in the Research Portal that you believe breaches copyright or violates any law, please contact openaccess@qub.ac.uk. 


\title{
Towards a spectroscopically accurate set of potentials for heavy hydride laser cooling candidates: effective core potential calculations of $\mathrm{BaH}$
}

\author{
Keith Moore, Brendan M. McLaughlin, and Ian C. Lane ${ }^{a)}$ \\ School of Chemistry and Chemical Engineering, Queen's University Belfast, Stranmillis Road, Belfast BT9 5AG, \\ $U K$
}

(Dated: 28 March 2016)

\begin{abstract}
$\mathrm{BaH}$ (and its isotopomers) is an attractive molecular candidate for laser cooling to ultracold temperatures and a potential precursor for the production of ultracold gases of hydrogen and deuterium. The theoretical challenge is to simulate the laser cooling cycle as reliably as possible and this paper addresses the generation of a highly accurate $a b$ initio ${ }^{2} \Sigma^{+}$potential for such studies. The performance of various basis sets within the multi-reference configuration-interaction (MRCI) approximation with the Davidson correction (MRCI+Q) is tested and taken to the Complete Basis Set (CBS) limit. It is shown that the calculated molecular constants using a 46 electron Effective Core-Potential (ECP) and even-tempered augmented polarized core-valence basis sets (aug-pCVnZ-PP, $n=4$ and 5) but only including three active electrons in the MRCI calculation are in excellent agreement with the available experimental values. The predicted dissociation energy $\mathrm{D}_{e}$ for the $\mathrm{X}^{2} \Sigma^{+}$state (extrapolated to the CBS limit) is $16895.12 \mathrm{~cm}^{-1}(2.094 \mathrm{eV})$, which agrees within $0.1 \%$ of a revised experimental value of $<16910.6 \mathrm{~cm}^{-1}$, while the calculated $\mathrm{r}_{e}$ is within $0.03 \mathrm{pm}$ of the experimental result.
\end{abstract}

\section{INTRODUCTION}

Recently, it has been proposed ${ }^{1}$ that ultracold hydrogen atoms may be formed by the photo-dissociation of laser cooled hydrides, provided the energy of the fragmentation products is much smaller than the average thermal energy of the parents. At the limit of zero kinetic energy release, the velocities of the hydrogen atoms will match that of the parents, forming a Maxwellian velocity distribution corresponding to a much lower hydrogen atom temperature than the original molecular gas $\left(\mathrm{T}_{H}=\frac{T_{M H}}{m_{M H}}\right)$. Quantum chemical calculations of the transition dipoles and Franck-Condon (FC) factors, using the post Hartree-Fock methods Complete Active Space Self Consistent Field (CASSCF) ${ }^{2}$ and $\mathrm{MRCI}^{3}$, confirmed that $\mathrm{BaH}$ was a very good candidate for demonstrating this kinematic effect as the barium atom has a considerable mass and the molecular radical is amenable to laser cooling ${ }^{4}$. In the earlier study a very small (triple zeta quality) two electron basis set for barium was used with the inner 52 electrons replaced by an ECP. To improve agreement with the experimental energy levels of the atom, an $\ell$-independent Core-Polarization Potential (CPP) was added to the calculation. Although the resulting basis set simulated the reported experimental FC factors very well, there were clearly issues with the calculation of the ground state equilibrium bond length $\mathrm{r}_{e}$ (experimental value $\mathrm{e}^{5}$ 2.23188651(19) $\AA$ ) and the dissociation limit ${ }^{6-9}$. This paper attempts to produce a global, high-quality $a b$ initio potential for the $\mathrm{X}^{2} \Sigma^{+}$ground state of $\mathrm{BaH}$. The heavier familial hydrides $\mathrm{YbH}$ and $\mathrm{RaH}$ are also attractive precursors of ultracold hydrogen but, unlike $\mathrm{BaH}$, there is currently a lack of reliable theoretical or

\footnotetext{
a)Electronic mail: i.lane@qub.ac.uk
}

experimental data to confirm their suitability. Hydrogen forms the basis of many spectroscopic tests of fundamental symmetries ${ }^{10}$, for the evolution of the Universe ${ }^{11}$ and in metrology ${ }^{12,13}$ (for example, the measurement of the Rydberg constant ${ }^{14}$ and the proton radius $\left.{ }^{15}\right)$.

$\mathrm{BaH}$ is naturally a heavier cousin of $\mathrm{BeH}$, the simplest stable neutral open shell diatomic. BeH possesses just five electrons and has recently been the focus of two important papers ${ }^{16,17}$ regarding the simulation of its ground potential energy curve. The first ${ }^{16}$ was a detailed $a b i n i$ tio study using very large correlation consistent basis sets (aug-cc-pCVnZ) up to seven zeta $(n=7)$ in character (indeed so large that the MOLPRO ${ }^{18} a b$ initio package could not use the largest $\ell=7$ functions in the basis set and the effect of excluding them was determined by a separate calculation). When determining the static electron correlation beyond Hartree-Fock level, the CASSCF wavefunction included the excited $3 s$ and $3 p$ orbitals on the Be atom within the active space despite this being a single electronic state calculation. To avoid the sizeextensivity issues inherent in MRCI (for dynamic electron correlation) the $\mathrm{MR}-\mathrm{ACPF}^{19}$ (multi-reference averaged coupled-pair functional) method was used instead. The size-extensivity and basis set superposition errors (BSSE) were determined to both be around $1-2 \mathrm{~cm}^{-1}$ at the $n=7$ level of calculation. The calculations were then extrapolated to the CBS limit. Despite the meticulous care taken in producing this ab initio potential, the resulting equilibrium bond length deviated by $0.13 \mathrm{pm}$ from the spectroscopic value. While in excellent agreement for a typical $a b$ initio calculation, for a benchmark molecule it is a little disappointing that such a powerful calculation could not achieve a better agreement. Tremendous pains were then made ${ }^{16}$ to include a host of minor corrections to the potential including electron correlation beyond the MR-ACPF method (to a level equivalent to full CI), scalar relativistic effects and diagonal Born- 
Oppenheimer corrections (DBOC). However, the energy effect of each of these additional contributions (all calculated using much smaller basis sets than used in the MRCI calculation) was small and mutually cancelling, while the improvement in bond length was just $0.04 \mathrm{pm}$. Furthermore, the bond dissociation energy was $90 \mathrm{~cm}^{-1}$ higher than the best experimental data ${ }^{20}$ at that point $\left(17590 \pm 200 \mathrm{~cm}^{-1}\right)$, though smaller than the raw $a b$ initio result $\left(17699 \mathrm{~cm}^{-1}\right)$. The second paper ${ }^{17}$ concerned taking that experimental work by Le Roy et a ${ }^{20}$ and fitting a new analytical function to the spectroscopic data. Just $5 \%$ of the rotationless adiabatic potential has yet to be covered experimentally and still the quantum number of the highest bound vibrational state is unclear. Again great attention to detail was conducted in this study, including mass corrected $C_{6}, C_{8}$ and $C_{10}$ constants for the different hydrogen isotopes. Despite this, the question of whether $v=12$ is the highest vibrational level is still unresolved but the experimental $D_{e}$ is now within $1 \mathrm{~cm}^{-1}$ of the corrected $a b$ initio value. If confirmed by future experiments, this would be an amazing achievement for $a b$ initio quantum chemistry.

Given the difficulties in describing the relatively simple $\mathrm{BeH}$, the task of completing accurate potentials for the heavier system $\mathrm{BaH}$, that contains an additional 52 electrons, looks formidable. Furthermore, relativistic effects that were tiny in $\mathrm{BeH}$ are amplified in such a heavy hydride. However, 46 or even 54 of the electrons can be replaced with an ECP and many of the scalar relativistic effects can be included in that potential. The larger core was used by Lane ${ }^{1}$ and earlier by Preuss and co-workers ${ }^{6}$ but in both instances the predicted ground state bond length was calculated to be much too short using a triple zeta basis set. While Preuss and co-workers ${ }^{6,7}$ managed to achieve $1 \mathrm{pm}$ accuracy using a smaller $(4 s, 4 p, 2 d)$ basis set, such behavior is symptomatic of simple error correction. Furthermore, both $\mathrm{D}_{e}$ and $\omega_{e}$ were much lower ${ }^{6}$ than experimental estimates at that time. It should not be forgotten that, despite the short bond length, the 2-electron, CPP + ECP calculation of FC factors is sufficiently accurate ${ }^{1}$ to be useful for screening good laser cooling transitions. For accurate calculation of the molecular constants, however, the evidence points to a minimum ten-electron basis set for barium.

Adopting the smaller 46-electron ECP allows the introduction of core-valence interactions with the $5 s$ and $5 p$ electrons as well as providing an opportunity to adjust the number of electrons used in calculating static and dynamic electron correlation. The change in ECP increased the predicted $r_{e}$ value of Kaupp et al by a further $10 \mathrm{pm}$ but this value was now $4 \mathrm{pm}$ too high. Going one step further, Allouche et al ${ }^{8}$ introduced an $\ell$-dependent CPP into a CI calculation of the lowest electronic states of $\mathrm{BaH}$ using a triple zeta basis set. Although there were eleven valence electrons, only three were active in the CI step. The $\mathrm{X}^{2} \Sigma^{+}$state equilibrium bond length was still $3 \mathrm{pm}$ too large, but the agreement is better than Kaupp et al. ${ }^{7}$ In addition the molecular constants for all the excited states correlating the lowest five electronic states of barium were calculated. They also investigated the effect of including spin-orbit coupling in the computation and demonstrated it made no difference (at the $100 \mathrm{fm}$ level) to the ground state bond length and altered excited states by $<0.3 \mathrm{pm}$. However, the spinorbit model adopted was fairly basic and consequently the spin-orbit constants were up to $60 \%$ too large. The calculated dissociation energy, $\mathrm{D}_{e}$, of $2.04 \mathrm{eV}$ was significantly larger than the earlier value of $1.79 \mathrm{eV}$ recommended by Fuentealba ${ }^{6}$ et al from their pseudo-potential plus single and double configuration interactive (CISD) model, with an equilibrium bond distance of $2.3707 \AA$. We note that Fuentealba et $\mathrm{al}^{6}$ also obtained a value of $2.1 \mathrm{eV}$ for $\mathrm{D}_{e}$ using a pseudo-potential plus local spin density (LSD) approximation with an equilibrium bond distance of $2.4829 \AA$. That dissociation energy is in excellent agreement with a recent relativistic coupled-cluster (RCCSDT) value of $2.062 \mathrm{eV}$ by Skripnokov et $a l^{9}$. This is interesting in itself as such agreement between relativistic and essentially non-relativistic calculations is rather unexpected in such a heavy hydride. Despite the close agreement between the highlighted theoretical bond energies, they are all larger than the accepted ${ }^{21}$ experimental value.

Following its discovery in the visible emission spectra ${ }^{22}$ of the Group II hydrides by Eagle in 1909 the $E^{2} \Pi \rightarrow$ $\mathrm{X}^{2} \Sigma^{+} 0 \quad 0$ band in $\mathrm{BaH}$ was assigned by Schaafsma ${ }^{23}$ around the time that Watson began a series of spectroscopic studies on $\mathrm{BaH}$ recording ${ }^{24}$ the $(1-1)$ and $(2-2)$ $\mathrm{E}^{2} \Pi \rightarrow \mathrm{X}^{2} \Sigma^{+}$emission bands (Funke later ${ }^{25}$ added the non-diagonal (0 1) and (2 - 1) bands). Watson followed that work with the first spectra of the $\mathrm{B}^{2} \Sigma^{+} \rightarrow \mathrm{X}^{2} \Sigma^{+}$ transition $^{26}$ in the near infra-red, which revealed an exceptionally large value for the spin-rotation constant in the upper state. As this constant was determined to be negative, it suggested the presence of the lower lying $\mathrm{A}^{2} \Pi$ state that was formally identified by the group ${ }^{27,28}$ two years later. Also conjectured in these papers was the presence ${ }^{27}$ of an even lower lying excited ${ }^{2} \Delta$ state, though this $\mathrm{H}^{2} \Delta$ potential was finally directly observed ${ }^{29}$ by Fabre et al. only in 1987. In 1936 Grunström $^{30}$ reported a new higher lying electronic state and analysed the $\left(\begin{array}{ll}0 & 0\end{array}\right)$ and $\left(\begin{array}{l}0 \\ -1\end{array}\right)$ bands of this violet $\mathrm{C}^{2} \Sigma^{+} \rightarrow \mathrm{X}^{2} \Sigma^{+}$ transition, a similar band having been observed in lighter Group II hydrides. Further investigation ${ }^{31}$ revealed that the $v^{\prime}=1$ level is abruptly predissociated above $N=10$ and it was speculated there was a lower bound state with an extended equilibrium bond length.

A series of papers in the 1960s by Kopp and colleagues revealed absorptions in the $\mathrm{UV}^{32}$, identified the $\mathrm{D}^{2} \Sigma^{+}$ state $^{21}$ perturbing the $\mathrm{C}^{2} \Sigma^{+}$vibronic structure, recorded the $\mathrm{B}^{2} \Sigma^{+} \leftarrow \mathrm{X}^{2} \Sigma^{+}$transition in $\mathrm{BaD}^{33}$ and added additional vibrational levels to the $\mathrm{A}^{2} \Pi \leftarrow \mathrm{X}^{2} \Sigma^{+}$absorption spectra $^{34}$ for both hydrogen isotopes. However, due to the highly diagonal transitions in the $\mathrm{A}^{2} \Pi, \mathrm{B}^{2} \Sigma^{+}$and $\mathrm{C}^{2} \Sigma^{+} \leftarrow \mathrm{X}^{2} \Sigma^{+}$absorption bands, direct observations of vibrational levels were limited to $v=0-2$ for all the 
electronic states involved. Only the $\mathrm{D}^{2} \Sigma^{+}$state had a potential minimum significantly displaced from that of the ground state but has only been studied in absorption so while the $\mathrm{D}^{2} \Sigma^{+}$state has been studied up to $v$ $=9$, the ground state remains unexplored ${ }^{5,35}$ above $v=$ 3. Furthermore, the often quoted experimental value for the dissociation energy in the ground state, $\mathrm{D}_{e}<16350$ $\mathrm{cm}^{-1}$, is based ${ }^{21}$ on the analysis of predissociation in the $\mathrm{C}^{2} \Sigma^{+}$state and assumptions made about the nature of mechanism involved.

\section{AB INITIO CALCULATIONS}

\section{A. Computational methods}

The initial results reported here were obtained using a parallel version of the MOLPRO ${ }^{18}$ suite of $a b$ initio quantum chemistry codes (release MOLPRO 2010.1). MRCI calculations based on State-Averaged Multi-Configuration-Self-Consistent-Field (SA-MCSCF) wavefunctions ${ }^{2,36,37}$ were conducted to determine the potential energy curves as a function of internuclear distance $r$ out to bond separation of $r=40$ bohr $\left(\mathrm{a}_{0}\right)$. When dealing with dissociating systems, size-consistency in the calculation is a particular concern, particularly with CI methods ${ }^{38}$ restricted to single and double excitations such as the MRCI code in MOLPRO. To estimate the missing higher excitations, the a posteriori Davidson correction ${ }^{39}$ was applied to all the results $(\mathrm{MRCI}+\mathrm{Q})$. To model the molecular orbitals a variety of correlation-consistent Gaussian basis sets ${ }^{40}$ were trialled; aug-cc-pVnZ, cc-pCVnZ-PP, cc-pwCVnZ-PP and aug-cc-pCVnZ-PP. In each calculation, the 46 core electrons of the barium atom were described with an effective core potential of the form ECP46MDF from the Stuttgart group ${ }^{41}$ leaving the outer ten $5 \mathrm{~s} 5 \mathrm{p} 6 \mathrm{~s}$ electrons. This allows one to test how each basis set performs for the molecular constants, e.g.; the equilibrium bond length and the dissociation energy $\mathrm{D}_{e}$ while keeping the active space constant.

All the electronic structure computations are performed in the $\mathrm{C}_{2 v}$ Abelian point group symmetry. In the $\mathrm{C}_{2 v}$ point group, molecular orbitals are labelled according to symmetries $a_{1}, b_{1}, b_{2}$ and $a_{2}$; when the molecular symmetry is reduced from $\mathrm{C}_{\infty v}$ to $\mathrm{C}_{2 v}$, the correlating relationships are $\sigma \rightarrow a_{1}, \pi \rightarrow\left(b_{1}, b_{2}\right), \delta \rightarrow\left(a_{1}, a_{2}\right)$. The preferred active space consists of 11 electrons and 17 molecular orbitals $7 a_{1}, 5 b_{1}, 3 b_{2}, 2 a_{2}$; i.e. a $(7,5,3,2)$ model. However, to describe the Rydberg character of the highly excited states of $A_{1}$ symmetry it was necessary sometimes to expand the active space to $\left(9 a_{1}, 5 b_{1}\right.$, $\left.3 b_{2}, 2 a_{2}\right)$. The averaging process was carried out on the lowest five ${ }^{2} \Sigma^{+},{ }^{2} \Pi$ and ${ }^{2} \Delta$ molecular states of this hydride.

\section{B. Ab initio potentials using an AV6Z basis}

Typically, the basis set of choice for an MRCI + Q calculation including excited electronic states is the augmented correlation consistent type such as aug-cc-pVnZ to provide the diffuse Gaussians required to model the higher energy electron orbitals. In addition, the larger the value of $n$ the more faithful the model wavefunctions match the real ones. Therefore, the first computations on this hydride were performed using an aug-cc-pV6Z basis on both atoms (Fig. 1), extending the previous work of Lane $^{1}$. The calculated potentials can be grouped according to three broad types:

(1) Bound potentials based on the interaction between a $\mathrm{Ba}^{+}$cation and $\mathrm{H}^{-}$anion e.g. the $\mathrm{X}^{2} \Sigma^{+}$and $\mathrm{A}^{2} \Pi$ states,

(2) Rydberg states with a single electron held within the potential of a $\mathrm{BaH}^{+}$molecular ion composed of $\mathrm{Ba}^{2+}$ and $\mathrm{H}^{-}$ions e.g. $\mathrm{C}^{2} \Sigma^{+}$and

(3) extended bond length states formed by the avoided crossing of largely repulsive valence curves and attractive ionic potentials e.g. $\mathrm{D}^{2} \Sigma^{+}$.

Only doublet states were investigated as the lowest quartet states ${ }^{8}$ are all repulsive. The historical spectroscopic assignments were used for the electronic states in Fig. 1 although it is clear that the adiabatic $3^{2} \Sigma^{+}$potential is reported as two separate states experimentally $\left(\mathrm{C}^{2} \Sigma^{+}\right.$ and $\mathrm{D}^{2} \Sigma^{+}$). The calculated energy gaps at the majority of the avoided crossings in $\mathrm{BaH}$ are tiny (the case of the $2^{2} \Sigma^{+}-\mathrm{X}^{2} \Sigma^{+}$adiabats being a noteworthy exception) and consistent with experiments where, for example, the Rydberg $\mathrm{C}^{2} \Sigma^{+}$vibrational progression ${ }^{21}$ is maintained for energies well above that of the $\mathrm{C} / \mathrm{D}^{2} \Sigma^{+}$states' closest approach (inset, Fig. 1). These AV6Z $\Lambda \mathrm{S} \Sigma$ potentials are consistent with previous work by Allouche ${ }^{8}$ et al.

For the ground $\mathrm{X}^{2} \Sigma^{+}$state the calculated value of $r_{e}$ $=2.18 \AA$ is in close agreement to the value of $r_{e}=2.16 \AA$ obtained $^{1}$ using a barium triple zeta basis set (cc-pV5Z basis on the hydrogen), a 56-electron ECP and an $\ell$ independent core polarisation potential (CPP). However, the accepted experimental value ${ }^{5}$ for $r_{e}$ is $2.2319 \AA$. The AV6Z basis set also predicts a dissociation energy $\mathrm{D}_{e}$ of $2.2687 \mathrm{eV}\left(18293.08 \mathrm{~cm}^{-1}\right)$ while the experimental value reported by Kopp et $a l^{21}$ is considerably smaller, $<2.0271 \mathrm{eV}\left(16350 \mathrm{~cm}^{-1}\right)$. Both the discrepancies in the equilibrium bond length and the dissociation energy are rather large for an MRCI+Q calculation with such a substantial barium basis set. Therefore, the investigation was widened to include a variety of other basis sets in an attempt to improve the agreement between the $a b$ initio results for the ground state for this hydride and the spectroscopic constants.

\section{Augmented basis sets}

An even tempered augmentation can be used to extend both the cc-pCVQZ-PP and the cc-pCV5Z-PP basis ${ }^{40}$ 


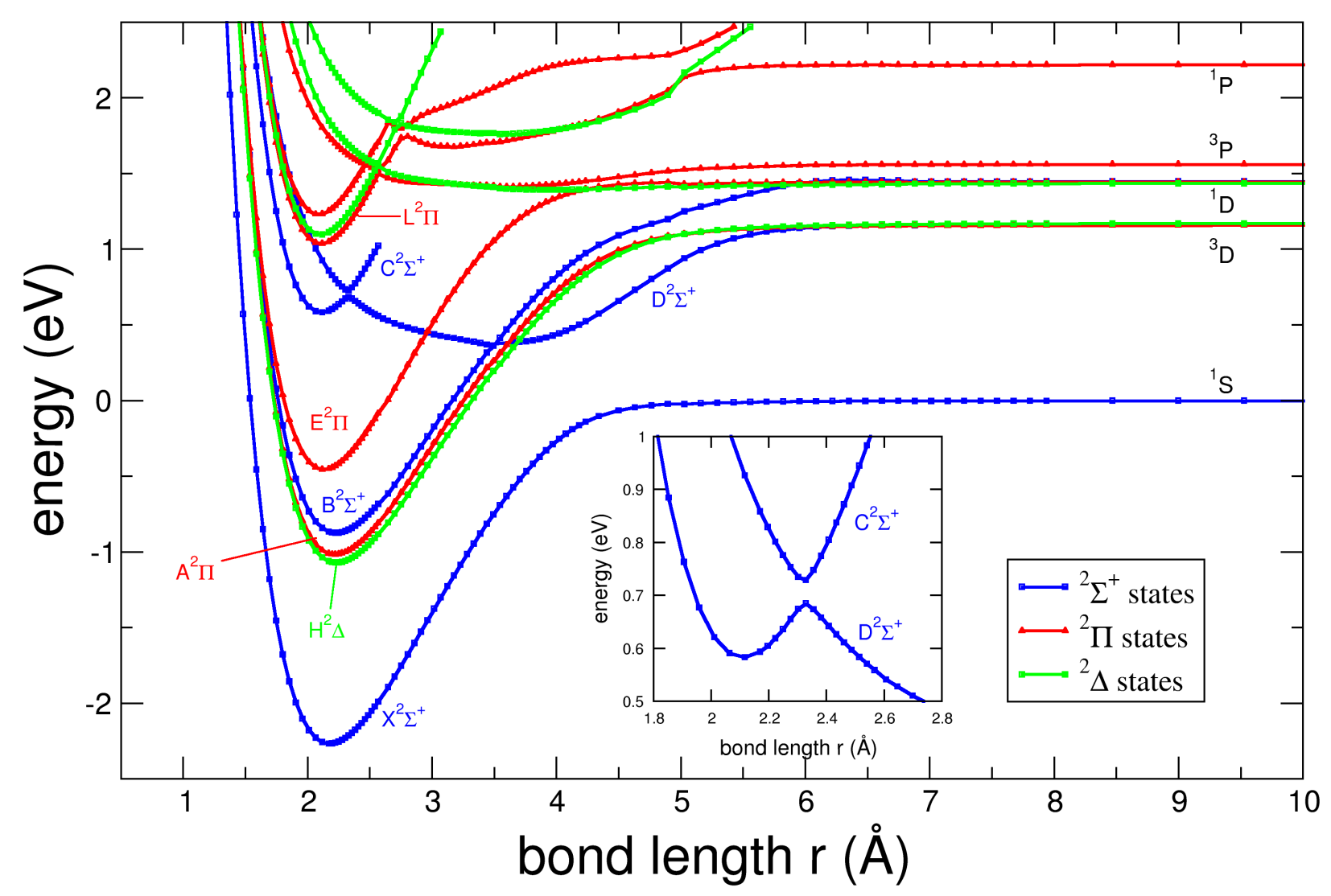

FIG. 1. MRCI $+Q$ results for the lowest 5 states of each symmetry $\left({ }^{2} \Sigma^{+},{ }^{2} \Pi\right.$ and $\left.{ }^{2} \Delta\right)$ as a function of bond length. The calculations have been performed with an aug-cc-pV6Z basis set and an ECP with the MOLPRO suite. The potentials shown were used to extract values for both the equilibrium bond distance $r_{e}$ and dissociation energy $\mathrm{D}_{e}$. For the ground $\mathrm{X}^{2} \Sigma^{+}$state, the calculated equilibrium bond length is $r_{e}=2.18 \AA$ while the dissociation energy is $\mathrm{D}_{e}=2.2687$ eV. The electronic states of the $\mathrm{Ba}$ atom fragments are also labelled (only $\mathrm{H}$ atoms in the lowest $1^{2} \mathrm{~S}$ state are produced at these energies). Inset: details of the avoided crossing between $\mathrm{C} / \mathrm{D}^{2} \Sigma^{+}$states.

sets. Each basis set is augmented with a single diffuse Gaussian function, the augmented exponent $\alpha^{\prime}$ given by the expression,

$$
\alpha^{\prime}(\ell)=\frac{\alpha^{2}(\ell)}{\beta(\ell)}
$$

where $\alpha(\ell)$ and $\beta(\ell)$ are respectively the last and second last exponent of the appropriate basis set. Calculations are performed in the MRCI+ Q approximation for the even tempered augmented sets (the additional functions are presented in Table I) and explicitly optimized augmented cc-pCVnZ-PP $(n=4$ and 5$)$ sets $^{40}$ (these explicitly optimized functions are presented in Table II). A large augmented basis set is chosen because in quantum chemistry calculations they are known to recover typically $\sim 99 \%$ or more of the electron correlation energy.

\section{D. $\operatorname{CCSD}(\mathrm{T})$ versus $\mathrm{MRCI}+\mathrm{Q}$}

In the search for an ideal basis set, numerous calculations with the MOLPRO suite were performed for the ground state of this hydride. Table III presents results for the different basis sets used in the MRCI+Q approximation, both for the equilibrium bond distance $r_{e}$ and dissociation energy $\mathrm{D}_{e}$, and compares them to $\operatorname{CCSD}(\mathrm{T})$ calculations $^{40}$ on $\mathrm{BaH}$. $\mathrm{CCSD}(\mathrm{T})$ is currently regarded as the most reliable theoretical method for information on ground state potentials. In the MRCI+Q calculations, the CASSCF step is performed over the lowest three ${ }^{2} A_{1},{ }^{2} B_{1}$, and ${ }^{2} A_{2}$ states while only the lowest 
TABLE I. Augmented functions to the cc-pCVnZ-PP basis set $^{40}$ for barium. The even tempered augmentation proceedure is used, where $\alpha(\ell)$ and $\beta(\ell)$ are respectively the last and second last exponent in appropriate basis set.

\begin{tabular}{|c|c|}
\hline cc-pCVQZ-PP & Barium \\
\hline Angular momentum function & Exponent $^{a}$ \\
\hline$s$ & 0.32620 \\
\hline$p$ & 0.10943 \\
\hline$d$ & 0.18940 \\
\hline$f$ & 0.43550 \\
\hline$g$ & 0.47145 \\
\hline cc-pCV5Z-PP & Barium \\
\hline Angular momentum function & Exponent $^{a}$ \\
\hline$s$ & 0.18570 \\
\hline$p$ & 0.11590 \\
\hline$d$ & 0.29510 \\
\hline$f$ & 0.38060 \\
\hline$g$ & 0.46480 \\
\hline$h$ & 0.62250 \\
\hline
\end{tabular}

${ }^{a}$ Estimated using $\alpha^{\prime}(\ell)=\frac{\alpha^{2}(\ell)}{\beta(\ell)}$

TABLE II. Augmented functions to the cc-pCVnZ-PP basis set for barium. For the augmentation proceedure the exponents are optimised ${ }^{40}$ on the energy of the ground state of the $\mathrm{BaH}$ molecule.

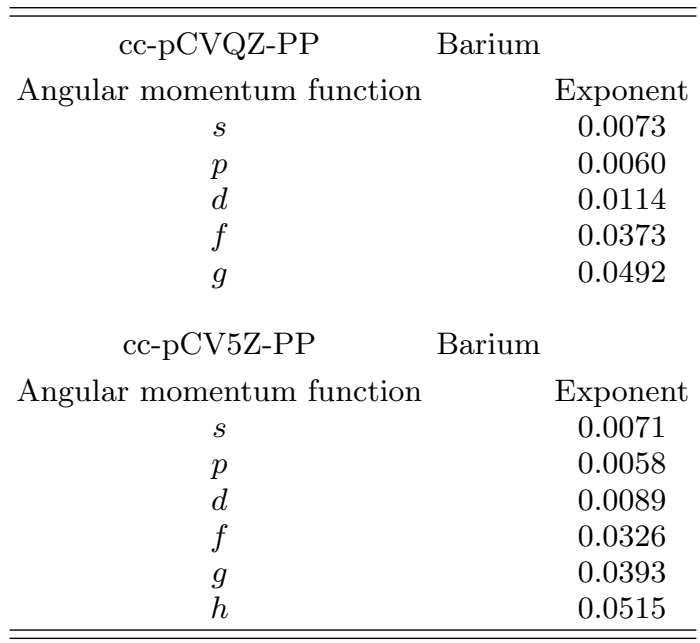

${ }^{2} A_{1}$ state appears in the MRCI code. It appears that the even tempered augmented basis set aug-cc-pCV5Z$\mathrm{PP}$ performs best at MRCI+Q level for the ground state, matching within $0.1 \mathrm{pm}$ the experimental $r_{e}$ value. The explicitly optimized augmented basis set aug-pCV5Z-PP gives comparable equilibrium bond distances. In contrast to calculations using $\mathrm{CCSD}(\mathrm{T})$, the cc-pCVnZ-PP basis sets pull the equilibrium bond lengths to much shorter values when using the MRCI $+\mathrm{Q}$ method. Augmenting the basis set with diffuse orbitals helps to counteract this effect. From Table III the CCSD(T), all electron, work ${ }^{40}$

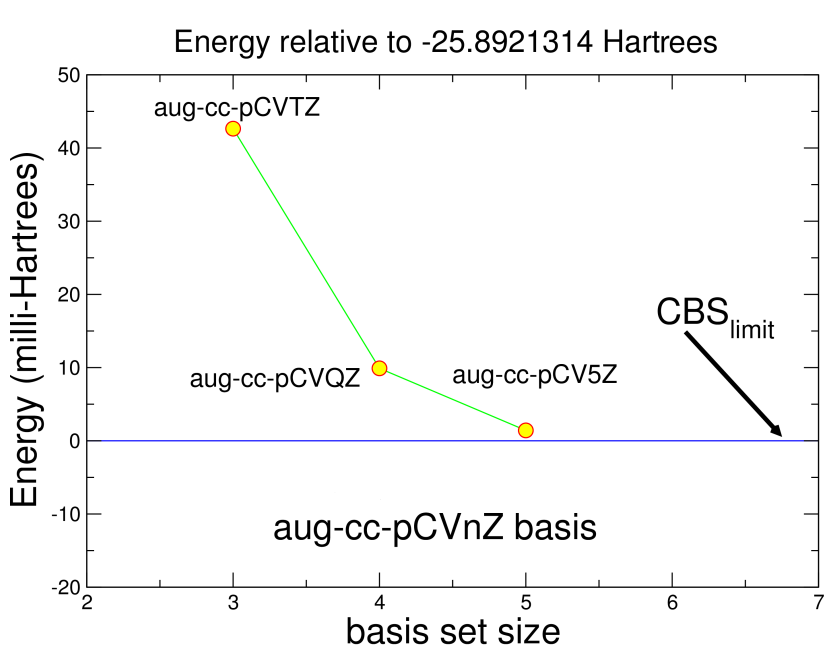

FIG. 2. BaH minimum energy in $\mathrm{X}^{2} \Sigma^{+}$state, the approach to the CBS limit using an active 3-electron model in the $\mathrm{MRCI}+\mathrm{Q}$ approximation. Even tempered aug-cc-pCVnZ-PP basis sets are used in the calculations.

provides results (with either the cc-pCV5Z-PP or ccpwCV5Z-PP basis) comparable to the present MRCI+Q results with the even tempered augmented basis set. The MRCI $+\mathrm{Q}$ calculations are also in excellent agreement with the previous $\mathrm{RCCSDT}^{9}$ and $\mathrm{MRCI}+\mathrm{Q}+$ including spin-orbital coupling $(\mathrm{SOC})^{42}$ values for the dissociation energy. In addition, this optimal basis set can also be used to carry out calculations of excited states, such as $\mathrm{A}^{2} \Pi$, a significant advantage of the MRCI method over $\operatorname{CCSD}(\mathrm{T})$.

\section{E. Complete Basis Set Limit}

To remove the uncertainty that any close agreement with experiment is the result of computational artefacts, it is best to extrapolate the energies $\mathrm{E}_{n}$ calculated with the $\mathrm{VnZ}$ basis sets to the limit of an infinite number of Gaussian functions. For the various basis sets shown in Table III the potential energy of the ground state via the complete basis set limit (CBS) is found using the formulae derived by Jensen ${ }^{43}$ with modifications by Karton and Martin $^{44}$

$$
E_{C B S}=E_{n}+\frac{\left(E_{n}-E_{n-1}\right)}{\frac{n}{n+1} \exp [9(\sqrt{n}-\sqrt{n-1})-1]}
$$

where here $n=5$. This expression is used to determine all the CBS potential energy curves in this study. Fig 2 shows the orderly approach to the CBS energy limit for the even tempered augmentation of the cc-pCVnZ-PP basis $^{40}$ set. While this extrapolation is usually adopted for static-correlation only, the small dynamic correlation found in the $\mathrm{BaH}$ calculations prompted its use here. The 
TABLE III. Spectroscopic constants for the $\mathrm{X}^{2} \Sigma^{+}$ground state of the BaH molecule: the equilibrium bond distance $r_{e}(\AA)$ and the dissociation energies $\mathrm{D}_{e}$ in $\mathrm{eV}$ and $\mathrm{cm}^{-1}$. Comparison of the present MRCI $+\mathrm{Q}$ results with a variety of other reported values using correlation - consistent basis sets ${ }^{40}$ (cc-pVnZ-PP, aug-cc-pVnZ-PP, cc-pCVnZ-PP, cc-pwCVnZ-PP, and aug-ccpCVnZ-PP), both with CI and CCSDT methods (fc, is frozen core, all, is all-electron) and other previous theoretical work. The best experimental values are also included.

\begin{tabular}{|c|c|c|c|c|}
\hline Basis & Method & $r_{e}(\AA)$ & $\mathrm{D}_{e}(\mathrm{eV})$ & $\mathrm{D}_{e}\left(\mathrm{~cm}^{-1}\right)$ \\
\hline cc-pVQZ-PP & $\mathrm{MRCI}+\mathrm{Q}$ & 2.2039 & 2.2229 & 17929.31 \\
\hline cc-pV5Z-PP & $\mathrm{MRCI}+\mathrm{Q}$ & 2.2329 & 2.5961 & 20939.44 \\
\hline CBS & $\mathrm{MRCI}+\mathrm{Q}$ & 2.2376 & 2.6580 & 21438.71 \\
\hline aug-cc-pV6Z & $\mathrm{MRCI}+\mathrm{Q}$ & 2.1800 & 2.2687 & 18293.08 \\
\hline cc-pCVQZ-PP & $\mathrm{MRCI}+\mathrm{Q}$ & 2.2367 & 2.0543 & 16569.43 \\
\hline cc-pCV5Z-PP & $\mathrm{MRCI}+\mathrm{Q}$ & 2.2151 & 2.5829 & 20832.97 \\
\hline CBS & $\mathrm{MRCI}+\mathrm{Q}$ & 2.2118 & 2.6715 & 21547.60 \\
\hline cc-pwCVQZ-PP & $\mathrm{MRCI}+\mathrm{Q}$ & 2.2367 & 2.0587 & 16604.92 \\
\hline cc-pwCV5Z-PP & $\mathrm{MRCI}+\mathrm{Q}$ & 2.2118 & 2.5954 & 20933.80 \\
\hline CBS & $\mathrm{MRCI}+\mathrm{Q}$ & 2.2080 & 2.6854 & 21659.71 \\
\hline aug-pCVQZ-PP & $\mathrm{MRCI}+\mathrm{Q}^{a}$ & 2.2354 & 2.0814 & 16787.75 \\
\hline aug-pCV5Z-PP & $\mathrm{MRCI}+\mathrm{Q}^{a}$ & 2.2332 & 2.0871 & 16833.78 \\
\hline CBS & $\mathrm{MRCI}+\mathrm{Q}^{a}$ & 2.2322 & 2.0946 & 16895.12 \\
\hline aug-pCVQZ-PP & $\mathrm{MRCI}+\mathrm{Q}^{b}$ & 2.2273 & 2.1157 & 17064.66 \\
\hline aug-pCV5Z-PP & $\mathrm{MRCI}+\mathrm{Q}^{b}$ & 2.2204 & 2.2384 & 18054.33 \\
\hline CBS & $\mathrm{MRCI}+\mathrm{Q}^{b}$ & 2.2187 & 2.2278 & 17968.83 \\
\hline cc-pVQZ-PP & $\operatorname{CCSD}(T), \mathrm{fc}^{c}$ & 2.3302 & 2.0311 & 16382.30 \\
\hline cc-pV5Z-PP & $\operatorname{CCSD}(\mathrm{T}), \mathrm{fc}^{c}$ & 2.3304 & 2.0563 & 16585.56 \\
\hline cc-pCVQZ-PP & $\operatorname{CCSD}(\mathrm{T}), \mathrm{fc}^{c}$ & 2.3266 & 2.0494 & 16529.91 \\
\hline cc-pCV5Z-PP & $\operatorname{CCSD}(T), \mathrm{fc}^{c}$ & 2.3280 & 2.0632 & 16641.21 \\
\hline cc-pwCVQZ-PP & $\operatorname{CCSD}(T), \mathrm{fc}^{c}$ & 2.3279 & 2.0498 & 16533.13 \\
\hline cc-pwCV5Z-PP & $\operatorname{CCSD}(\mathrm{T}), \mathrm{fc}^{c}$ & 2.3282 & 2.0632 & 16641.21 \\
\hline cc-pCVQZ-PP & $\operatorname{CCSD}(T)$, all $^{c}$ & 2.2378 & 2.0298 & 16371.82 \\
\hline cc-pCV5Z-PP & $\operatorname{CCSD}(\mathrm{T})$, all $^{c}$ & 2.2316 & 2.0723 & 16714.61 \\
\hline cc-pwCVQZ-PP & $\operatorname{CCSD}(\mathrm{T}), \mathrm{all}^{c}$ & 2.2379 & 2.0355 & 16417.79 \\
\hline cc-pwCV5Z-PP & $\operatorname{CCSD}(\mathrm{T})$, all $^{c}$ & 2.2327 & 2.0736 & 16725.10 \\
\hline aug-cc-pVQZ & $\operatorname{RCCSD}(\mathrm{T})^{d}$ & 2.2400 & 2.0886 & 16846.08 \\
\hline aug-cc-pV5Z & $\mathrm{MRCI}+\mathrm{Q}+\mathrm{SOC}^{e}$ & 2.2417 & 2.0860 & 16825.11 \\
\hline \multirow[t]{3}{*}{ Triple-zeta } & CIPSI $(\mathrm{ECP}+\mathrm{CPP})^{f}$ & 2.2620 & 1.9700 & 15889.49 \\
\hline & Pseudo-potential + LSD $^{g}$ & 2.4280 & 2.1000 & 16938.03 \\
\hline & Pseudo-potential + CISD $^{g}$ & 2.3707 & 1.7900 & 14437.66 \\
\hline Experiment & & $2.2319^{h}$ & $<2.0271^{i}$ & $<16350^{j}$ \\
\hline cc-pCVQZ-PP & $\mathrm{MRCI}+\mathrm{Q}(3 \mathrm{e})^{k}$ & 2.23670 & 2.0543 & 16569.43 \\
\hline cc-pCVQZ-PP & $\mathrm{MRCI}+\mathrm{Q}(5 \mathrm{e})^{l}$ & 2.23725 & 2.1359 & 17227.59 \\
\hline
\end{tabular}

${ }^{a} \mathrm{MRCI}+\mathrm{Q}$, even tempered basis set augmentation functions, present work.

${ }^{b}$ MRCI+Q, explicitly energy optimized augmentation functions, present work.

${ }^{c} \operatorname{CCSD}(\mathrm{T})$, fc and $\operatorname{CCSD}(\mathrm{T})$, all : private communication from Peterson (2015).

${ }^{d}$ RCCSD(T), Skripnikov and co-workers ${ }^{9}$.

${ }^{e} \mathrm{MRCI}+\mathrm{Q}+\mathrm{SOC}$, Gao et $a l^{42}$.

${ }^{f}$ CIPSI Allouche ${ }^{8}$.

${ }^{g}$ Pseudo-potential + local spin density $(\mathrm{LSD})^{6}$, Pseudo-potential $+\mathrm{CISD}^{6}$.

${ }^{h}$ Experiment, Ram and Bernath ${ }^{5} .{ }^{i}$ Experiment, Kopp et $a l^{21} .{ }^{j}$ Assuming $\mathrm{Ba}\left({ }^{3} \mathrm{D}_{3}\right)$ limit.

${ }^{k} \mathrm{MRCI}+\mathrm{Q}$, active 3-electron model, present work. ${ }^{l} \mathrm{MRCI}+\mathrm{Q}$, active 5-electron model, present work. 
close agreement with the experimental $\mathrm{r}_{e}$ value found in the CBS potential supports this choice.

In Table III the results for the equilibrium bond distance $r_{e}$ and dissociation energy $\mathrm{D}_{e}$ from these CBS limit, MRCI+Q calculations are compared with the coupled cluster appromixation $\operatorname{CCSD}(T)^{40,45-47}$, in its various implementations and with other theoretical methods ${ }^{8,9,48}$. The density functional theory (DFT) work of Wang and Andrews ${ }^{48}$ using a 6-311++G $(3 d f, 3 p d)$ basis yielded a value of $2.2520 \AA$ and second order Møller Plesset perturbation theory gave 2.2440 $\AA^{48}$ respectively for the equilibrium bond distance. Gao et $a l^{42}$ have conducted calculations for the ground $\mathrm{X}^{2} \Sigma^{+}$ and the first excited state $A^{2} \Pi$ of this hydride at the MRCI+Q+SOC level. Their $a b$ initio results for the spectroscopic constants $r_{e}(2.2417 \AA)$ and $\mathrm{D}_{e}(2.0860 \mathrm{eV})$ of $\mathrm{BaH}\left(\mathrm{X}^{2} \Sigma^{+}\right)$ground state are similar to those obtained from the relativistic - coupled-cluster results ${ }^{9}$ (RCCSDT), respectively $2.24 \AA$ and $2.0840 \mathrm{eV}$, and in reasonable agreement with the available experimental results ${ }^{49}$. The other $a b$ initio results of great accuracy are those using $\operatorname{CCSD}(\mathrm{T})$ where the cc-pCV5Z-PP (all electron) result is within $0.02 \mathrm{pm}$ of the experimental value ${ }^{5}$ but if extrapolated to the CBS limit this agreement will be somewhat poorer. Using an aug-cc-pCVnZ/CBS $\mathrm{MRCI}+\mathrm{Q}$ calculation the $a b$ initio equilibrium bond distance here is within $0.031 \mathrm{pm}$ of the best spectroscopic value.

\section{MODELLING THE GROUND STATE}

\section{A. Spectroscopic constants of the ground state}

An important requirement is ensuring that the final potential behaves correctly at all interatomic distances, particularly at long-range where dispersion forces are dominant ${ }^{50}$. In atomic units, the asymptotic atom-atom potential has the form ${ }^{51}$

$$
V(r) \simeq V_{\infty}-\left(\frac{C_{6}}{r^{6}}+\frac{C_{8}}{r^{8}} \ldots\right)
$$

where $C_{6}, C_{8}$ etc. are the polarizabilities with $V_{\infty}$ the asymptotic limit (atomic products). $r$ is the atom-atom internuclear separation. The dynamic polarization of the ground state, which allows the computation of the leading $C_{6}$ term (148 a.u.), has been calculated ${ }^{52}$ by Derevianko et al. To calculate the following $C_{8}$ term, the formula derived by Tang ${ }^{53}$ requires the quadrupole polarizabilities but there are two theoretical values ${ }^{54,55}$ for Ba that produce $C_{8}$ values that differ by more than $60 \%$. The smaller value, 11242 a.u., is consistent with the corresponding term calculated ${ }^{56}$ for the neighbouring hydride CsH (11710 a.u.), and is therefore the value recommended at present. There is no theoretical or experimental value at present for $C_{10}$ in $\mathrm{BaH}$ so the expansion was truncated at $C_{8}$ : the corresponding calculated value ${ }^{56}$ in $\mathrm{CsH}$ is $1.194 \times 10^{6}$ a.u.

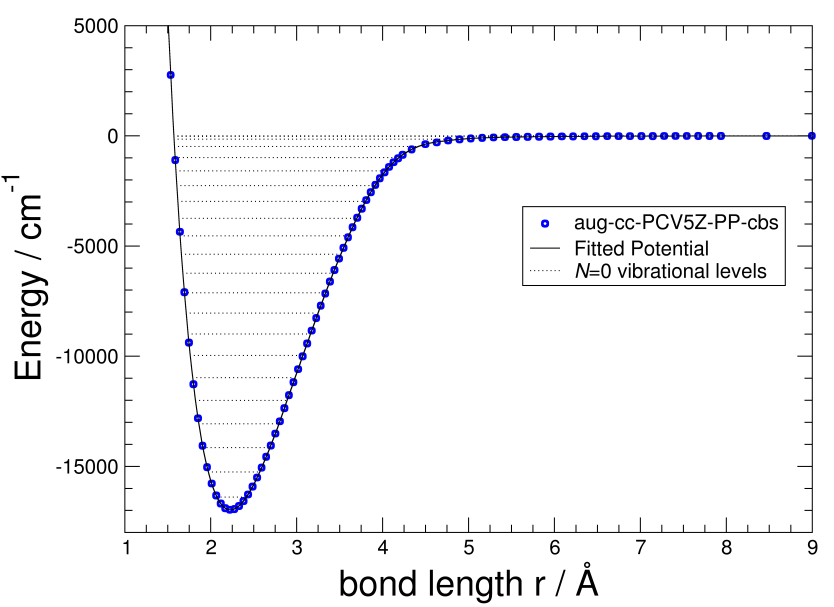

FIG. 3. Analysis of $a b$ initio $\mathrm{X}^{2} \Sigma^{+}$ground state of BaH using LEVEL $^{57}$. The $a b$ initio data was taken with the aug-cc-pCVnZ basis sets ( $n=4$ and 5$)$ and extrapoliated to the CBS limit. The vibrational levels found in the fit are also presented.

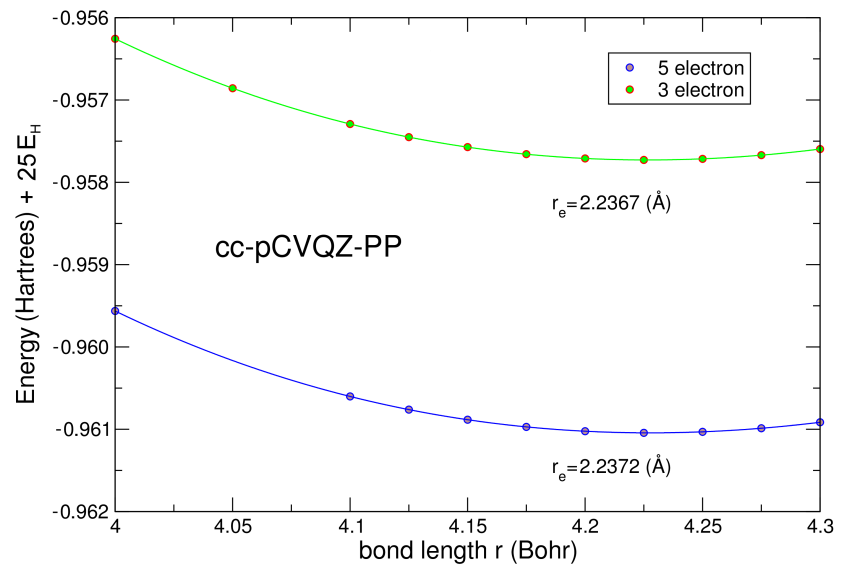

FIG. 4. Comparison of the ground state energy for the $\mathrm{BaH}$ molecule in the region of the equilibrium bond distance using both an active 3-electron and 5-electron model in the MRCI+Q approximation. The cc-pCVQ-PP basis set ${ }^{40}$ is used for both calculations. The absolute energies are adjusted by $25 \mathrm{E}_{H}$.

The LEVEL ${ }^{57}$ program (Version 8.2 ) was used to calculate the rovibrational levels of the resulting $a b$ initio $\mathrm{X}^{2} \Sigma^{+}$aug-cc-pCVnZ/CBS potential. A smooth potential was produced through interpolation of the $a b$ initio points with a 4-point piecewise polynomial (NUSE $=4$, IR2=1), and extrapolation past $r=15.88 \AA$ using a fixed $C_{6}=7.110512 \times 10^{5} \mathrm{~cm}^{-1} \AA^{-6}\left(\right.$ no $C_{8}$ or $\left.C_{10}\right)$ $(\mathrm{ILR}=2)$. Based on this potential the equilibrium bond length and dissociation energy were determined to be 
TABLE IV. Vibrational term energies in the $\mathrm{X}^{2} \Sigma^{+}$ground state of the BaH molecule. Comparison of the present MRCI+Q results with the aug-cc-pCVnZ-PP even tempered basis set taken to the CBS limit and experimental data. All energies in $\mathrm{cm}^{-1}$.

\begin{tabular}{rrrrrr}
\hline \hline$v^{\prime \prime}$ & MRCI & Relative value $^{a}$ & Experimental $^{5}$ & $\mathrm{RKR}^{b}$ & $\Delta \mathrm{E} \mathrm{RKR}$ \\
\hline 0 & 581.3491 & 0 & 0 & 580.53 & 0.8191 \\
1 & 1722.2738 & 1140.9247 & 1139.2896 & 1719.84 & 2.4338 \\
2 & 2832.9237 & 2251.5746 & 2249.6062 & 2830.15 & 2.7737 \\
3 & 3915.9012 & 3334.5521 & 3331.1192 & 3911.46 & 4.4412 \\
4 & 4981.7132 & 4400.3641 & & 4963.77 & 17.9432 \\
5 & 6024.9686 & 5443.6195 & & 5987.08 & 37.8886 \\
6 & 7029.1845 & 6447.8354 & & 6981.39 & 47.7945 \\
7 & 8006.0703 & 7424.7212 & & 7946.70 & 59.3703 \\
8 & 8957.6993 & 8376.3502 & & & \\
9 & 9878.6102 & 9297.2611 & & & \\
10 & 10772.2217 & 10190.8726 & & & \\
11 & 11633.1528 & 11051.8037 & & & \\
12 & 12461.4104 & 11880.0613 & & & \\
13 & 13257.3637 & 12676.0146 & & & \\
14 & 14016.6614 & 13435.3123 & & & \\
15 & 14729.2373 & 14147.8882 & & & \\
16 & 15386.3161 & 14804.9670 & & & \\
17 & 15966.4205 & 15385.0714 & & & \\
18 & 16436.4095 & 15855.0604 & & & \\
19 & 16749.4237 & 16168.0746 & & & \\
20 & 16882.9819 & 16301.6328 & & & \\
21 & 16894.9472 & 16313.5981 & & & \\
\hline \hline
\end{tabular}

${ }^{a} \mathrm{E}(\mathrm{MRCI})-\mathrm{E}($ experimental $)=\Delta \mathrm{E}\left(v^{\prime}-v^{\prime \prime}\right) \mathrm{cm}^{-1}:(1-0)=+1.64 ;(2-1)=+0.33 ;(3-2)=+1.46$.

${ }^{b}$ Based on experimental data prior ${ }^{58}$ to 1982.

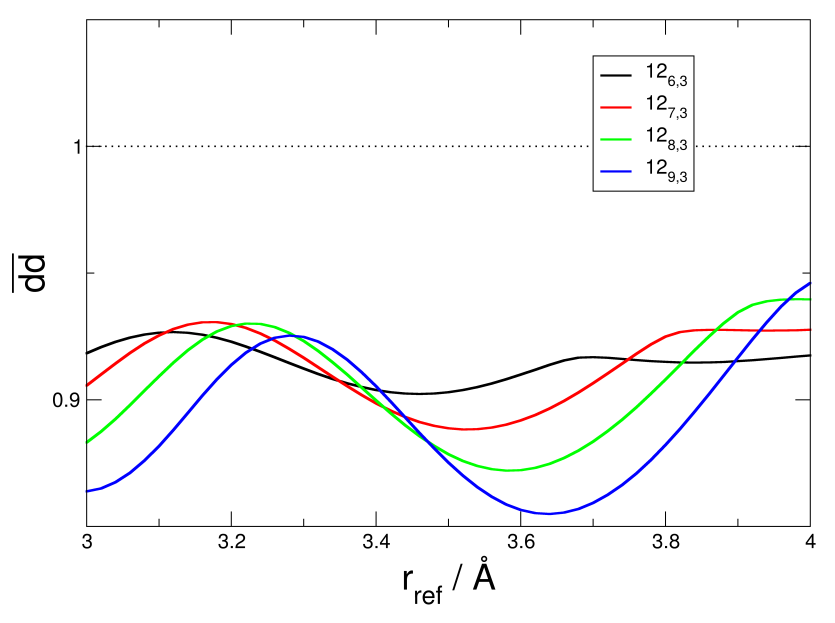

FIG. 5. Tuning $\mathrm{r}_{r e f}$ to improve the fitting of the $a b$ initio $\mathrm{X}^{2} \Sigma^{+}$ground state of $\mathrm{BaH}$ using betaFIT $\mathrm{T}^{59}$.

$2.2322 \AA$ and $16895 \mathrm{~cm}^{-1}$. Twenty-two vibrational levels were predicted for the system, with $v=21$ lying just 0.2 $\mathrm{cm}^{-1}$ below the dissociation limit. Comparison of the relative energies of the first four vibrational levels based on this potential with experimental values ${ }^{5}$ show good agreement, all within $1.7 \mathrm{~cm}^{-1}$ (Table IV). There is a significant jump in the error with respect to the earlier RKR levels at $v=4$, a result of the lack of experimental data for the higher vibrational levels and consequently the present results are probably more reliable for those missing vibrational levels.

\section{B. 3 electron versus 5 electron $\mathrm{MRCI}+\mathrm{Q}$}

Though the calculated bond length is only $<0.031 \mathrm{pm}$ away from the experimental value, another effort was made to explore possible improvements to the accuracy of the $a b$ initio potential. In order to quantify the effect of including additional dynamic electron correlation, further MRCI+Q calculations were performed with the cc-pCVQZ-PP basis set ${ }^{40}$ using both 3 - and 5-electrons active at the CI stage. From the $a b$ initio work shown in Fig. 4 it is found that the active 5-electron model lowers the absolute (total) energy of the ground state minimum for this hydride by just 3 milli-Hartrees $\left(\mathrm{mE}_{h}\right)$. The subsequent change in the equilibrium bond distance $r_{e}$ was also tiny, an increase of $0.055 \mathrm{pm}$. These very small changes in the total energy and equilibrium distance subsequently justifies the use of the 3-electron model for both the ground and excited states considered as opposed 
to the more computationally intensive active 5-electron model.

\section{Including spectroscopic data}

There is an extensive selection of pair-potentials in the literature to describe the $r$ dependence of the interaction energy in a diatomic molecule (see the supplementary information from Xie et al for a comprehensive list ${ }^{60}$ with over a hundred examples). Two of the most successful functions are the Tang-Toennies ${ }^{61}$ potential (and its variants) and the Morse-Long-Range ${ }^{62}$ (MLR) function. The $a b$ initio data here was fitted to a MLR potential using betaFIT (version 2.1) ${ }^{59}$ in order to find an analytical function to express the potential. Prior to fitting, the potential was shifted by $0.03 \mathrm{pm}$ so that the $a b$ initio potential minimum was aligned with the experimental $\mathrm{r}_{e}$ value to an accuracy better than $0.01 \mathrm{pm}$. This simplifies the inclusion of experimental data at a later stage. The MLR potential is described by

$$
V_{\mathrm{MLR}}(r) \equiv \mathfrak{D}_{e}\left(1-\frac{u(r)}{u\left(r_{e}\right)} e^{-\beta(r) y_{p}^{r_{e}}(r)}\right)^{2}
$$

where $\mathfrak{D}_{e}$ is the dissociation energy and $u\left(r_{e}\right)$ is value of $u(r)$ at the equilibrium bond distance.

Both $\mathrm{V}_{\mathrm{MLR}}$ and $\beta(r)$ are expanded in terms of two internuclear distances $\mathrm{r}_{e}$ and $\mathrm{r}_{r e f}$, according to the radial variable

$$
y_{n}^{r_{i}}(r)=\frac{r^{n}-r_{i}^{n}}{r^{n}+r_{i}^{n}}
$$

where $\mathrm{r}_{i}=\mathrm{r}_{e}$ or $\mathrm{r}_{r e f}$ and the latter is usually greater than or equal to $r_{e}$. As the fit is viable for a large range of $\mathrm{r}_{r e f}$, it is worth tuning this value to improve the fit (Fig. 5). MLR functions producing local minima were selected as starting points for later fitting to spectral data. Typically, two different values of $n$ are required for an acceptable fit (in the present work, $n=8$ and 3 ) in the $\beta(r)$ expansion. The first issue is what to set as the error in the ab initio points. Since the vibrational frequencies are calculated to within an average of $2 \mathrm{~cm}^{-1}$ the error in each $a b$ initio point was initially set rather arbitrarily as $10 \mathrm{~cm}^{-1}\left(5 \times 10^{-5} \mathrm{E}_{h}\right)$. However, Dattani and Le Roy ${ }^{63}$ have shown that even in the $6 \mathrm{e} \mathrm{Li}_{2}$ molecule a deviation in $\mathrm{D}_{e}$ of up to $68 \mathrm{~cm}^{-1}$ still persists in the calculated $\mathrm{b}^{1} \Pi$ state. Therefore, an increased error of $20 \mathrm{~cm}^{-1}$ was adopted as a compromise value. The final analytical potential is reported as $\operatorname{MLR}_{p, q}^{r_{r e f}}\left(N_{\beta}\right)$, where $p$ and $q$ are the two values of $n$ required and consists of $N_{\beta}$ expansion coefficients. A similar ${ }^{64}$ potential (replacing an earlier version $^{65}$ that used only one expansion co-efficient $p$ ) has recently been used to describe the ground state of $\mathrm{MgH}$, the only hydride other than $\mathrm{H}_{2}$ with an experimental dissociation energy determined to sub-wavenumber accuracy. The fitted $\mathfrak{D}_{e}$ is just over $4 \mathrm{~cm}^{-1}$ lower than the result from the $a b$ initio potential (Table VI), as it was allowed to vary slightly during the fitting process.
The parameters fitted to $\mathrm{MLR}_{8,3}^{3.34}$ (12) from the $a b$ initio potential were then transferred to DPotFit ${ }^{66,67}$ (version 2.0) and combined with $\mathrm{B}^{2} \Sigma^{+} \rightarrow \mathrm{X}^{2} \Sigma^{+}$ emission $^{26,28,33}$ and $\mathrm{X}^{2} \Sigma^{+}$infrared data ${ }^{35}$. The earlier emission data was quoted to $0.01 \mathrm{~cm}^{-1}$ while the stated resolution of the infrared data was $0.005 \mathrm{~cm}^{-1}$.

While $\mathrm{r}_{\text {ref }}=3.34 \AA$ does not correspond to a local minimum in Fig. 5, those MLRs obtained from the local minima in betaFIT were not found to be as successful. $p=8, q=3$ were selected as the best parameters after screening other combinations. As DPotFit utilizes a dimensionless root-mean-square-deviation $(\overline{d d})$ as its means of determining how closely a model matches the observed data, it is crucial that a reasonable assessment of the uncertainty in the observable data is made.

$$
\overline{d d}=\sqrt{\frac{1}{N_{\text {data }}} \sum_{i=1}^{N_{\text {data }}}\left(\frac{E_{\text {calc }}(i)-E_{\text {obs }}(i)}{u_{\text {obs }}(i)}\right)^{2}}
$$

Thus, as the uncertainty in the $a b$ initio $\mathrm{E}_{\text {obs }}$ was set to $20 \mathrm{~cm}^{-1}$, deviations from the fitted MLR do not lead to a large increase in the $\overline{d d}$. At the chosen $\mathrm{r}_{r e f}$ the $\overline{d d}$ between the $\operatorname{MLR}_{8,3}^{3.34}(12)$ potential fitted by betaFIT and the $a b$ initio points was only 0.4570 representing a close fit within the selected uncertainty. By contrast the original emission data was quoted to much higher accuracy, so any deviations between the model and the spectral measurements can lead to a significant increase (left hand data, Table VI) in $\overline{d d}$. Due to the arbitrary nature of the uncertainty assignment in the $a b$ initio data, the associated $\overline{d d}$ should not be compared directly to the $\overline{d d}$ in fits to experimental data.

As DPotFit tries to fit to very accurate spectroscopic data, the initial uncertainty in the fit is very high. Allowing $\beta$ parameters to become fitted parameters produces a final potential which matches the experimental data much more closely than the $a b$ initio potential alone. During the fitting, both $C_{6}$ and $C_{8}$ were fixed at the recommended theoretical values, and were incorporated as the long range tail of the MLR potential with Douketistype damping ${ }^{68}$ with $s=-1$. Additionally, the effect of spin rotation coupling is incorporated through a modification to the MLR potential ${ }^{67}$ using the expansion coefficients listed in Table VII.

As the considered spectral data covers a range of isotopomers of $\mathrm{BaH}$ (electronic transitions of ${ }^{138} \mathrm{BaH}$ and ${ }^{138} \mathrm{BaD}$; infrared transitions of ${ }^{138} \mathrm{BaH},{ }^{137} \mathrm{BaH}$, $\left.{ }^{136} \mathrm{BaH},{ }^{135} \mathrm{BaH}\right)$, all of which have very small values of reduced mass, it is valuable to consider the impact of the Born-Oppenheimer breakdown $(\mathrm{BOB})^{69}$ corrections. DPotFit incorporates BOB corrections through the atom-dependant potential, with the effects parametrised within the radial strength functions:

$$
\tilde{S}_{\mathrm{ad}}^{\mathrm{A}}(r)=y_{p_{\mathrm{ad}}}^{e q}(r) u_{\infty}^{\mathrm{A}}+\left[1-y_{p_{\mathrm{ad}}}^{e q}(r)\right] \sum_{i=0}^{N_{\mathrm{ad}}^{\mathrm{A}}} u_{i}^{\mathrm{A}} y_{p_{\mathrm{ad}}}^{e q}(r)^{i}
$$


TABLE V. Summary of the spectral data used when fitting with DPotFit. A total of $1769 \mathrm{~B}^{2} \Sigma-\mathrm{X}^{2} \Sigma$ transition and $409 \mathrm{X}^{2} \Sigma^{+}$ IR emission lines were incorporated.

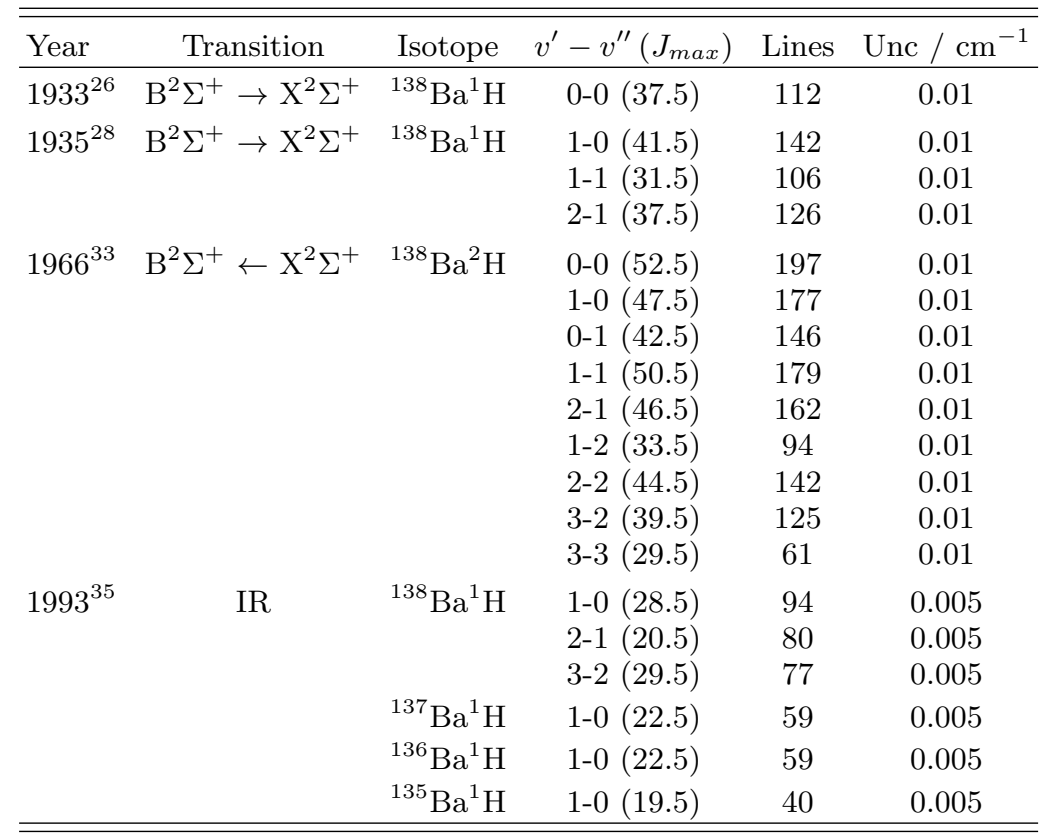

TABLE VI. The $\operatorname{MLR}_{p, q}^{r_{r e f}}\left(N_{\beta}\right)$ potential parameters obtained through a fit to the ab initio potential, and after initial optimisation with spectral data using DPotFit. All energies in $\mathrm{cm}^{-1}$.

\begin{tabular}{|c|c|c|c|c|c|c|c|}
\hline \multicolumn{4}{|c|}{$\operatorname{MLR}_{8,3}^{3.34}(12), a b$ initio only } & \multicolumn{4}{|c|}{$\operatorname{MLR}_{8,3}^{3.34}(12)$, ab initio + spectra } \\
\hline & \multicolumn{3}{|c|}{$\begin{array}{cl}\mathfrak{D}_{e} & 16891.5947 \\
\mathrm{r}_{e} & 2.2319 \AA \\
d d & 266.447\end{array}$} & \multicolumn{4}{|c|}{$\begin{array}{rl}\mathfrak{D}_{e} & 16841.8651 \\
\frac{\mathrm{r}_{e}}{d d} & 2.2318 \AA \\
& 6.083\end{array}$} \\
\hline$\beta_{0}$ & 0.114040 & & -87.820760 & $\beta_{0}$ & 0.096691 & & -86.606654 \\
\hline$\beta_{1}$ & -7.619529 & $\beta_{8}$ & -171.874803 & $\beta_{1}$ & -7.616896 & $\beta_{8}$ & -154.070717 \\
\hline$\beta_{2}$ & -17.121381 & $\beta_{9}$ & -5.387603 & $\beta_{2}$ & -16.467554 & $\beta_{9}$ & 34.3428882 \\
\hline$\beta_{3}$ & -19.233860 & $\beta_{10}$ & 256.820563 & $\beta_{3}$ & -16.902752 & $\beta_{10}$ & 324.143391 \\
\hline$\beta_{4}$ & 3.673494 & $\beta_{11}$ & 260.900999 & $\beta_{4}$ & 6.565879 & $\beta_{11}$ & 334.502110 \\
\hline$\beta_{5}$ & 44.305094 & & 83.391642 & $\beta_{5}$ & 44.183256 & $\beta_{12}$ & 116.057107 \\
\hline$\beta_{6}$ & 31.801460 & & & $\beta_{6}$ & 28.715007 & & \\
\hline
\end{tabular}

$$
\tilde{R}_{\mathrm{na}}^{\mathrm{A}}(r)=y_{p_{\mathrm{na}}}^{e q}(r) t_{\infty}^{\mathrm{A}}+\left[1-y_{p_{\mathrm{na}}}^{e q}(r)\right] \sum_{i=0}^{N_{\mathrm{na}}^{\mathrm{A}}} t_{i}^{\mathrm{A}} y_{p_{\mathrm{ad}}}^{e q}(r)^{i}
$$

Where $\tilde{S}_{\text {ad }}^{\text {A }}(r)$ is the atom-dependant adiabatic BOB radial strength function and $\tilde{R}_{\text {na }}^{\mathrm{A}}(r)$ the non-adiabatic BOB radial strength function. Incorporating fitted terms ( $\mathrm{Ta}-$ ble VII) for $N_{\text {ad }}=N_{\text {na }}=2$ along with fitted MLR parameters (Table VI) while keeping $r_{e}$ fixed at $2.2319 \AA$ produced an MLR potential with $\overline{d d}=6.143$. Allowing $r_{e}$ to also be a fitted parameter lowered $\mathfrak{D}_{e}$ by nearly $50 \mathrm{~cm}^{-1}$ but further reduced $\overline{d d}$ to 6.083 . While substantially higher than what would usually be deemed a "good" fit ${ }^{67}(\overline{d d}<1)$, comparison with results from the most recent study ${ }^{5}$ of the $\mathrm{E}\left({ }^{2} \Pi\right) \rightarrow \mathrm{X}\left({ }^{2} \Sigma^{+}\right)$transition, a data set not used in the DPotFit analysis, highlights the much improved agreement with the experimental observations (Table VIII) than the raw ab initio results from Table IV.

In addition, a number of further details were evident while developing the hybrid potential. The experimental values $^{35}$ for the spin-rotation constant $\gamma(v)$ follow a very clear $v$ dependence ${ }^{70,71}$ (Fig. 6) that can be modelled as a simple Taylor (linear) expansion:

$$
\gamma(v)=\gamma_{0}+\gamma_{1}\left(v+\frac{1}{2}\right)
$$

with $\gamma_{0}=0.19456 \mathrm{~cm}^{-1}$ and $\gamma_{1}=-4.9813 \times 10^{-3} \mathrm{~cm}^{-1}$. Finally, the experimental dissociation energy quoted in the literature has its origin in the sudden onset of rapid predissociation in the $\mathrm{C}^{2} \Sigma^{+} v^{\prime}=1, N^{\prime}=10$ rovibronic 


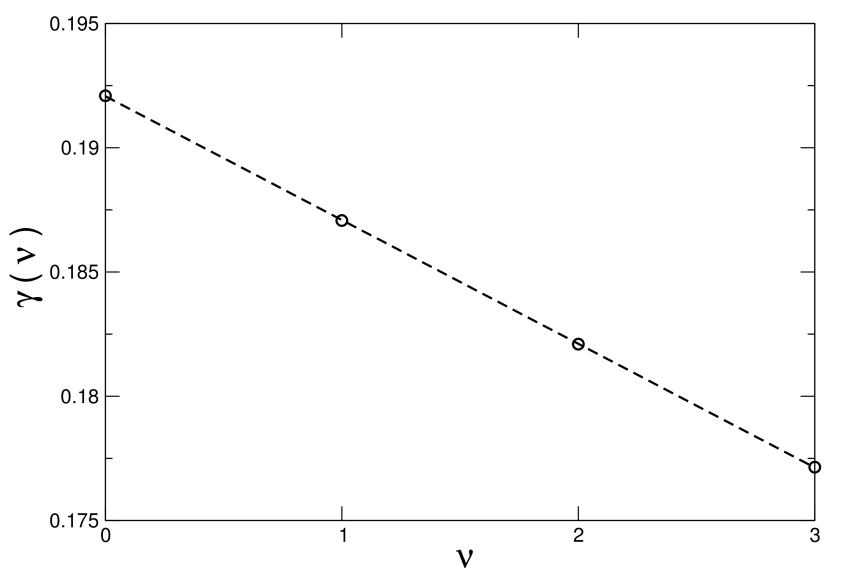

FIG. 6. The $v$ dependence of the experimental spin-rotation constants $\gamma$ from the experimental work ${ }^{35}$ of Walker, Hedderich and Bernath.

TABLE VII. Additional parameters defining the ${ }^{2} \Sigma^{+}$spinrotation coupling and the BOB corrections in the final MLR potential, obtained after initial optimisation with spectral data using DPotFit.

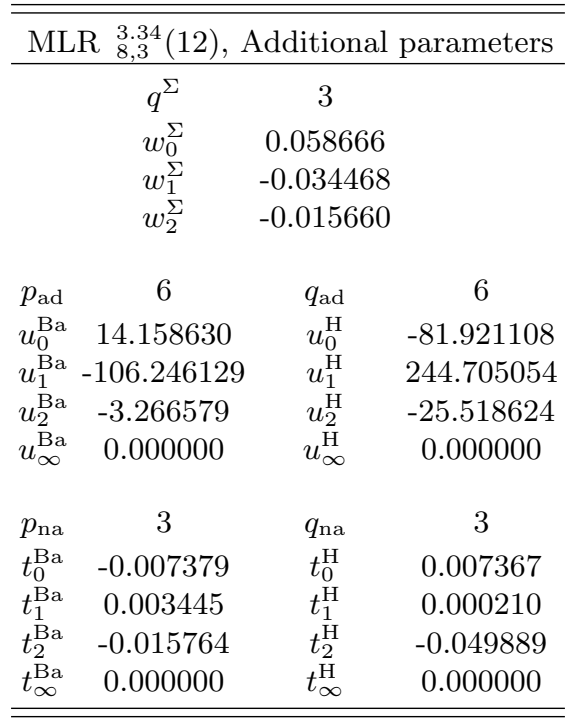

level. This level lies $25942.6 \pm 0.5 \mathrm{~cm}^{-1}$ above the $\mathrm{X}^{2} \Sigma^{+}$ minimum. Assuming that this indicates the dissociation limit of the (diabatic) $\mathrm{D}^{2} \Sigma^{+}$potential lies above $\mathrm{C}^{2} \Sigma^{+}$ $v^{\prime}=1, N^{\prime}=9\left(25874.6 \pm 0.3 \mathrm{~cm}^{-1}\right)$ and identifying the asymptote as belonging to $\mathrm{Ba}\left({ }^{3} \mathrm{D}_{1}\right)$ and not $\mathrm{Ba}\left({ }^{3} \mathrm{D}_{3}\right)$, this would fix the dissociation energy $\mathrm{D}_{e}$ of the ground state between $16842.6 \mathrm{~cm}^{-1}$ and $16910.6 \mathrm{~cm}^{-1}$ which is consistent with the present $a b$ initio value and other recent theoretical $^{9}$ results, while the DPotFit result is within $1 \mathrm{~cm}^{-1}$ of the lower limit. It is therefore tentatively suggested that the correct dissociation limit for the $\mathrm{D}^{2} \Sigma^{+}$

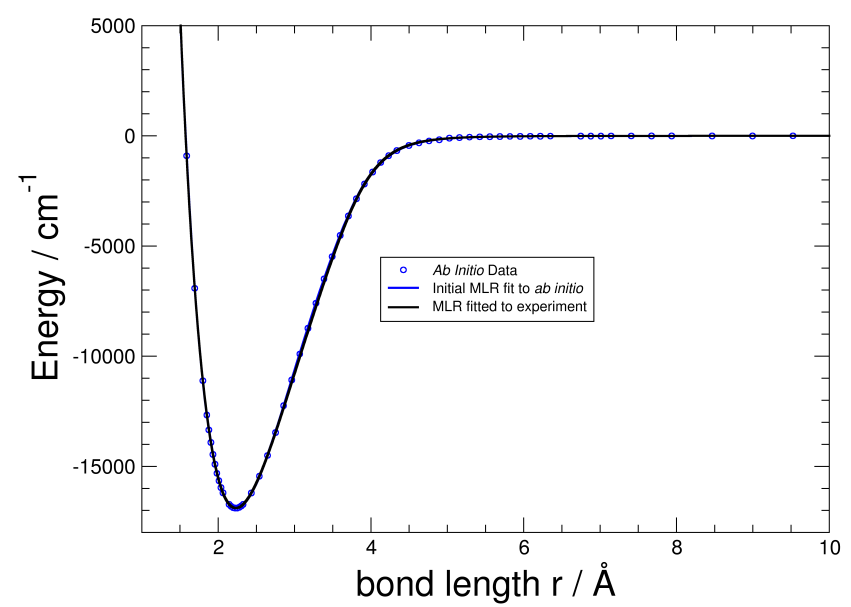

FIG. 7. $\operatorname{MLR}_{8,3}^{3.34}(12)$ fit to $a b$ initio data points, and final $\operatorname{MLR}_{8,3}^{3.34}(12)$ fit to experimental data.

state is actually $\mathrm{Ba}\left({ }^{3} \mathrm{D}_{1}\right)+\mathrm{H}\left({ }^{2} \mathrm{~S}_{\frac{1}{2}}\right)$ and at long range ought to be labelled $\mathrm{D} \frac{1}{2}(1)$ in Hund's case (c).

\section{Excited states}

Extending the $a b$ initio study to excited states, the lowest energy ${ }^{2} \Pi$ state was calculated using the even tempered augmented cc-pCV5Z-PP basis set ${ }^{40}$. Despite being carried out with a CASSCF calculation including $5 A_{1}, 5 B_{1}$ and $5 A_{2}$ states and the MRCI being carried out over $5 B_{1}$ states, the resulting potential has an $\mathrm{r}_{e}$ of $2.2698 \AA$, within $0.38 \mathrm{pm}$ of the best experimental value from Barrow (quoted in Allouche ${ }^{8}$ et al). The calculated difference in $\mathrm{X}^{2} \Sigma^{+}$and $\mathrm{A}^{2} \Pi$ bond lengths is therefore $\Delta \mathrm{r}_{e}$ $=3.97 \mathrm{pm}$ compared to the experimental difference of $4.16 \mathrm{pm}$, a discrepancy of just $2 \mathrm{~mA}$. Of course, for a truly faithful reproduction of the $\mathrm{A}^{2} \Pi$ state not only must spin-orbit coupling be included in the calculation, but also the angular momentum coupling with other electronic states. However, for $\mathrm{FC}$ factors the fact that $\Delta \mathrm{r}_{e}$ is so close to experiment is reassuring and small energy shifts in general do not have a large effect.

The two potentials were then used to obtain FC factors between the $\mathrm{X}^{2} \Sigma^{+}$and $\mathrm{A}^{2} \Pi$ vibronic states using LEVEL. The highly diagonal nature of the $\mathrm{A}^{2} \Pi \leftarrow \mathrm{X}^{2} \Sigma^{+}$transition is clear when the wavefunctions from both states are compared as in Fig. 8. The diagonal $\mathrm{FC}$ factor $f_{00}$ is almost identical to that found in the earlier study by Lane $^{1}$ using the larger ECP and a much smaller triple zeta quality basis set, though the agreement reduces with each increase in vibrational quantum number (by $f_{33}$ the difference is $5.8 \%$, compared to $0.1 \%$ for $f_{00}$ ). Using the revised dissociation energy $\mathrm{D}_{e}$ of the ground state $\left(<16910.6 \pm 0.5 \mathrm{~cm}^{-1}\right)$, the ${ }^{3} \mathrm{D}-{ }^{1} \mathrm{~S}$ separation in bar- 
TABLE VIII. Vibrational term energies in the $\mathrm{X}^{2} \Sigma^{+}$ground state of the $\mathrm{BaH}$ molecule using DPotFit. Comparison of the vibrational levels from the present combined $a b$ initio/spectroscopic potential and experimental ${ }^{5}$ data independent of fit. All energies in $\mathrm{cm}^{-1}$.

\begin{tabular}{rrrrrr}
\hline \hline$v$ & $\begin{array}{c}\text { Experiment } \\
(\text { Ram } \text { et al) }\end{array}$ & $\begin{array}{c}\text { DPotFit }^{a} \\
r_{e} \text { fix }\end{array}$ & \multicolumn{1}{c}{$\begin{array}{c}\Delta \mathrm{E} \\
r_{e} \text { fix }\end{array}$} & $\begin{array}{c}\text { DPotFit }^{b} \\
r_{e} \text { fit }\end{array}$ & \multicolumn{1}{c}{$\begin{array}{c}\Delta \mathrm{E} \\
r_{e} \text { fit }\end{array}$} \\
\hline 0 & 0.0000 & 0.0000 & 0.0000 & 0.0000 & 0.0000 \\
1 & 1139.2896 & 1139.2884 & -0.0012 & 1139.2897 & 0.0001 \\
2 & 2249.6062 & 2249.6084 & -0.0022 & 2249.6078 & -0.0016 \\
3 & 3331.1192 & 3331.1212 & -0.0020 & 3331.1234 & -0.0042 \\
\hline \hline
\end{tabular}

${ }^{a}$ Zero Point Energy $=580.6050 \mathrm{~cm}^{-1}$

${ }^{b}$ Zero Point Energy $=580.5910 \mathrm{~cm}^{-1}$

ium $\left(9372 \mathrm{~cm}^{-1}\right)$, the Term energy of the minimum of the $A^{2} \Pi$ potential (excluding spin-orbit separation) from Barrow $^{72}$ and co-workers and finally the $\mathrm{X}^{2} \Sigma^{+}$zero point energy, the experimental dissociation energy $\mathrm{D}_{e}$ of the $\mathrm{A}^{2} \Pi$ state is $<16001.3 \pm 2.0 \mathrm{~cm}^{-1}$ which is in good agreement with our calculated value of $16076.75 \mathrm{~cm}^{-1}$. This is further support for our proposed revision of the experimental value for the $\mathrm{BaH} \mathrm{X}{ }^{2} \Sigma^{+}$dissociation energy $\mathrm{D}_{e}$ to $<16910.6 \pm 0.5 \mathrm{~cm}^{-1}$.

Using the same basis set, ab initio calculations were performed in the MRCI+Q approximation for five states of each of the ${ }^{2} A_{1},{ }^{2} B_{1}$ and ${ }^{2} A_{2}$ symmetries for bond length out to $40 \mathrm{a}_{0}$. The results from all these calculations are presented in Fig. 9. The ${ }^{2} A_{1}$ states are the most common symmetry at lower energies and therefore valence states dominate in Fig. 9. Comparing to the previous AV6Z calculation, the less diffuse nature of the largest Gaussian functions in our adopted aug-pCVnZ$\mathrm{PP}$ basis sets has resulted in the greater weighting of valence states over Rydbergs and so the $\mathrm{C}^{2} \Sigma^{+}$state has disappeared. To describe the Rydberg character of the ${ }^{2} \Sigma^{+}$states, it's necessary to expand the active space in the MRCI+Q calculations to at least $\left(8 a_{1}, 5 b_{1}, 3 b_{2}, 2_{2}\right)$.

\section{SUMMARY AND CONCLUSION}

In this paper we have attempted to develop a reliable potential energy curve for the $\mathrm{X}^{2} \Sigma^{+}$state of the $\mathrm{BaH}$ radical. Encouraged by the close agreement in the literature between relativistic and non-relativistic $a b$ initio calculations, we have used the MRCI $+\mathrm{Q}$ method to determine the shape of the $\mathrm{X}^{2} \Sigma^{+}$potential and the dissociation energy. With an even-tempered augmented ccpCVnZ-PP basis set taken to the CBS limit, we have calculated the equilibrium bond length to within $0.031 \mathrm{pm}$ of the experimental value and of comparable accuracy

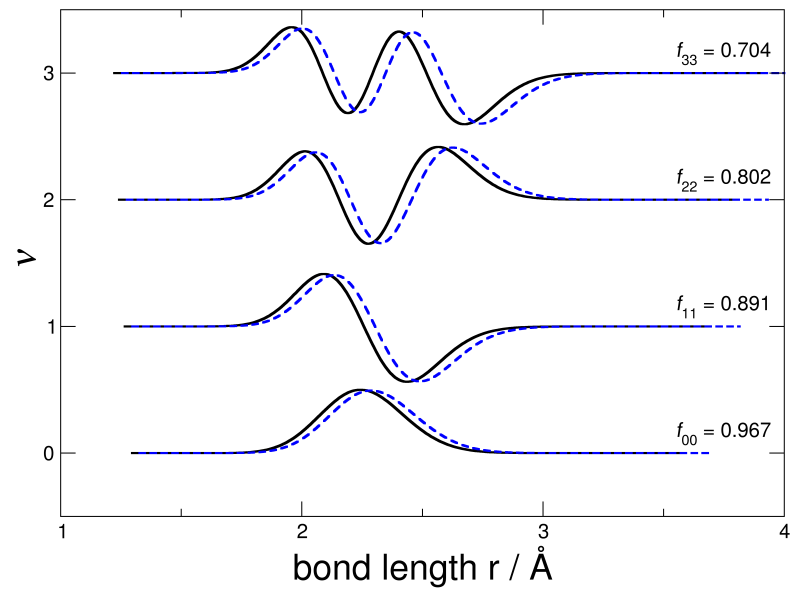

FIG. 8. The almost identical first four vibrational wavefunctions in the $\mathrm{X}$ and $\mathrm{A}$ states of $\mathrm{BaH}$. The larger equilibrium bond length displaces the excited state slightly but the overlap is sufficient to ensure diagonal Franck-Condon factors close to 1. Also labelled are the diagonal Franck-Condon (FC) factors $f_{v_{i} v_{i}}$.

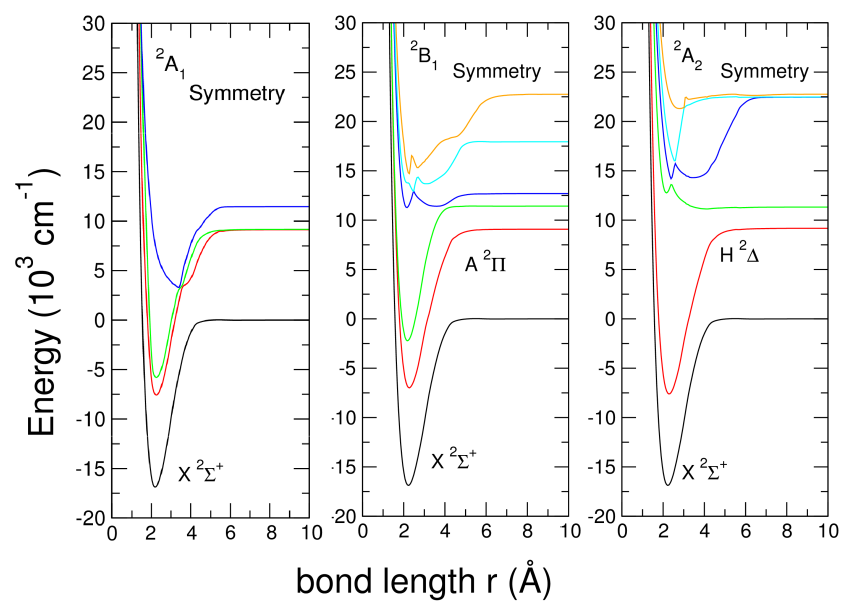

FIG. 9. Low lying states of $\mathrm{BaH}$ using an active 3-electron model in the MRCI $+\mathrm{Q}$ approximation. The even tempered aug-cc-pCV5Z-PP basis set is used for the calculations.

to the more trusted $\operatorname{CCSD}(\mathrm{T})$ technique. While the ten $5 s 5 p 6 s$ electrons from barium are necessary to calculate the static correlation energy, only the valence $6 s$ electrons are needed to cover the majority of the dynamic correlation. The computed dissociation energy $\left(16895.12 \mathrm{~cm}^{-1}\right)$ is in good agreement with other theoretical values suggesting a revision of the experimental dissociation energy to $\mathrm{D}_{e}<16910.6 \mathrm{~cm}^{-1}$. The calculated dissociation energy of the excited $\mathrm{A}^{2} \Pi$ state $\left(16076.75 \mathrm{~cm}^{-1}\right)$ is also 
consistent with this experimental value. The vibrational levels of the $a b$ initio $\mathrm{X}^{2} \Sigma^{+}$potential achieve an average agreement of just over $1 \mathrm{~cm}^{-1}$ with the best experimental values. Refining the potential with experimental data using DPotFit improves the agreement to better than $0.005 \mathrm{~cm}^{-1}$ for the lowest vibrational levels.

\section{ACKNOWLEDGMENTS}

I. C. L. thanks the Leverhulme Trust for financial support, a PDRA fellowship for B. M. M. and a Ph D studentship for K. M. under Research Grant RPG-2014-212. We would like to thank Professor K. Peterson for providing the explicitly optimized augmented functions for the barium atom for his cc-pCVnZ-PP basis sets and for many helpful discussions regarding this ab initio work. Grants of computational time at the National Energy Research Scientific Computing (NERSC) Center in Oakland, CA, USA and at the High Performance Computing Center Stuttgart (HLRS) of the University of Stuttgart, Stuttgart, Germany, where some of these computations were performed, are gratefully acknowledged.

${ }^{1}$ I. C. Lane, Phys. Rev. A 92, 022511 (2015).

${ }^{2}$ H. J. Werner and P. J. Knowles, Chem. Phys. Lett. 115, 259 (1985).

${ }^{3}$ H. J. Werner and P. J. Knowles, J. Chem. Phys. 89, 5803 (1988).

${ }^{4}$ M. G. Tarallo, G. Z. Iwata, and T. Zelevinsky, Phys. Rev. A 93, 032509 (2016).

${ }^{5}$ R. S. Ram and P. F. Bernath, J. Mol. Spec. 283, 18 (2013).

${ }^{6}$ P. Fuentealba, O. Reyes, H. Stoll, and H. Preuss, J. Chem. Phys. 87, 5338 (1987).

${ }^{7}$ M. Kaupp, P. Schleyer, H. Stoll, and H. Preuss, J. Chem. Phys. 94, 1360 (1991).

${ }^{8}$ A. R. Allouche, G. Nicolas, J. C. Barthelat, and F. Spiegelmann,

J. Chem. Phys. 96, 7646 (1992).

${ }^{9}$ L. V. Skripnikov, N. S. Mosyagin, and A. V. Titov, Chem. Phys. Lett. 555, 79 (2013).

${ }^{10}$ V. A. Kostelecky and A. J. Vargas, Phys. Rev. D 92, 056002 (2015).

${ }^{11}$ W. Ubachs, J. Koelemeij, K. Eikema, and E. Salumbides, J. Mol. Spectro. 320, 1 (2016).

${ }^{12}$ J. S. Rigden, Hist. Stud. Phys. Sci. 13, 335 (1983).

${ }^{13}$ H. Kragh, Hist. Stud. Phys. Sci. 15, 67 (1985).

${ }^{14}$ B. Cagnac, M. D. Plimmer, L. Julien, and F. Biraben, Rep. Prog. Phys. 57, 853 (1994).

${ }^{15}$ S. G. Karshenboim, Phys. Rev. A 91, 012515 (2015).

${ }^{16}$ J. Koput, J. Chem. Phys. 135, 244308 (2011).

${ }^{17}$ N. S. Dattani, J. Mol. Spectrosc. 311, 76 (2015).

${ }^{18}$ H.-J. Werner, P. J. Knowles, R. Lindh, F. R. Manby, and M. S. et. al., "MOLPRO 2010, http://www.molpro.net,".

${ }^{19}$ R. J. Gdanitz and R. Ahlrichs, Chem. Phys. Lett. 143, 413 (1988).

${ }^{20}$ R. J. Le Roy, D. R. Appadoo, R. Colin, and P. F. Bernath, J. Mol. Spectrosc. 236, 178 (2006).

${ }^{21}$ I. Kopp, N. Åslund, G. Edvinsson, and B. Lindgren, Ark. Fysik. 30, 321 (1966).

${ }^{22}$ A. Eagle, Astrophys. J. 30, 231 (1909)

${ }^{23}$ A. Schaafsma, Z. Phys. 74, 254 (1932).

${ }^{24}$ W. R. Fredrickson and W. W. Watson, Phys. Rev. 39, 753 (1932).

${ }^{25}$ G. Funke, Z. Phys. 84, 610 (1933).

${ }^{26}$ W. W. Watson, Phys. Rev. 43, 9 (1933).

${ }^{27}$ W. W. Watson, Phys. Rev. 47, 213 (1935).

${ }^{28}$ P. G. Koontz and W. W. Watson, Phys. Rev. 48, 937 (1935).
${ }^{29}$ G. Fabre, A. El-Hachimi, R. Stringat, C. Effantin, A. Bernard, J. dIncan, and J. Vergès, J. Phys. B: At. Mol. Phys. 20, 1933 (1987).

${ }^{30}$ B. Grunström, Z. Phys. 99, 595 (1936).

${ }^{31}$ G. W. Funke and B. Grunström, Z. Phys. 100, 293 (1936).

${ }^{32}$ G. Edvinsson, I. Kopp, B. Lindgren, and N. Åslund, Ark. Fysik. 25, 95 (1963)

${ }^{33}$ I. Kopp and R. Wirhed, Ark. Fysik. 32, 307 (1966).

${ }^{34}$ I. Kopp, M. Kronekvist, and A. Guntsch, Ark. Fysik. 32, 371 (1966).

${ }^{35}$ K. A. Walker, H. G. Hedderich, and P. F. Bernath, Mol. Phys. 78, 577 (1993).

${ }^{36}$ P. Siegbahn, A. Heiberg, B. Roos, and B. Levy, Phys. Scr. 21 , 323 (1980).

${ }^{37}$ H. J. Werner and P. J. Knowles, J. Chem. Phys. 82, 5053 (1985).

${ }^{38}$ L. Meissner, Chem. Phys. Lett. 146, 204 (1988).

${ }^{39}$ S. Langhoff and E. R. Davidison, Int. J. Quantum Chemistry 8 , 61 (1974)

${ }^{40} \mathrm{~K}$. Peterson, "private communication, all the basis sets are available from the Peterson group website, http://tyr0.chem.wsu.edu/ kipeters/basis.html save augcc-pCVnZ-PP," (2015).

${ }^{41}$ I. S. Lim, H. Stoll, and P. Schwerdtfeger, J. Chem. Phys. 124, 034107 (2006).

${ }^{42}$ Y. Gao and T. Gao, Phys. Rev. A 90, 052505 (2014).

${ }^{43}$ F. Jensen, Theo. Chem. Acc. 113, 267 (2005).

${ }^{44}$ A. Karton and J. M. L. Martin, Theo. Chem. Acc. 115, 330 (2006).

${ }^{45}$ J.Č́ízek, J. Chem. Phys. 45, 4256 (1966).

${ }^{46}$ G. D. Purvis III and R. J. Barlett, J. Chem. Phys. 76, 1910 (1982).

${ }^{47}$ J. D. Watts, J. Gauss, and R. J. Barlett, J. Chem. Phys. 98, 8718 (1993).

${ }^{48}$ X. Wang and L. Andrews, J. Phys. Chem. A 108, 11500 (2004).

${ }^{49}$ P. J. Linstrom and W. G. Mallard, "NIST Chemistry WebBook NIST Standard Reference Database Number 69," National Institute of Standards and Technology, Gaithersburg, MD, USA, http://www.webbook.nist.gov (2005).

${ }^{50}$ J. Mitroy, M. S. Safronova, and C. W. Clark, J. Phys. B: At. Mol. Opt. Phys. 43, 202001 (2010).

${ }^{51}$ R. Côte and A. Dalgarno, Phys. Rev 62, 012709 (2000).

${ }^{52}$ A. Derevianko, S. G. Porsev, and J. F. Babb, Atomic Data and Nuclear Data Tables 96, 323 (2010).

${ }^{53}$ K. T. Tang, Phys. Rev. 177, 108 (1969).

${ }^{54}$ S. H. Patil, Eur. Phys. J. D 10, 341 (2000).

${ }^{55}$ S. G. Porsev and A. Derevianko, JETP, 102, 195 (2006).

${ }^{56}$ S. H. Patil and K. T. Tang, J. Chem. Phys. 106, 2298 (1997).

${ }^{57}$ R. J. Le Roy, LEVEL 8.2 University of Waterloo Chemical Physics Research Report CP-668 (2014).

${ }^{58}$ M. Ramanaish and S. Lakishman, Physica C 113, 263 (1982).

${ }^{59}$ R. J. Le Roy, betafit University of Waterloo Chemical Physics Research Report CP-666 (2013).

${ }^{60}$ J. C. Xie, S. K. Mishra, T. Kar, and R.-H. Xie, Chem. Phys. Lett. 605, 137 (2014).

${ }^{61}$ K. T. Tang and J. P. Toennies, J. Chem. Phys. 80, 3726 (1984).

${ }^{62}$ R. J. Le Roy, Y. Y. Huang, and C. Jary, J. Chem. Phys. 125, 164310 (2006).

${ }^{63}$ N. S. Dattani and R. J. Le Roy, ArXiV , 1508.07184 (2015).

${ }^{64}$ R. Henderson, A. Shayesteh, J. Tao, C. Haugen, P. Bernath, and R. Le Roy, J. Phys. Chem. A 117, 133373 (2013).

${ }^{65}$ A. Shayesteh, R. D. E. Henderson, R. J. Le Roy, and P. F. Bernath, J. Phys. Chem. A 111, 12495 (2007).

${ }^{66}$ R. J. Le Roy, N. S. Dattani, J. A. Coxon, A. J. Ross, P. Crozet, and C. Linton, J. Chem. Phys. 131, 204309 (2009).

${ }^{67}$ R. J. Le Roy, DPotFit University of Waterloo Chemical Physics Research Report CP-667 (2013).

${ }^{68}$ C. Douketis, G. Scoles, S. Marchetti, M. Zen, and A. J. Thakkar, J. Chem. Phys. 76, 3057 (1982).

${ }^{69}$ R. J. Le Roy and Y. Huang, Journal of Molecular Structure (Theochem) 591, 175 (2002). 
${ }^{70}$ J. M. Brown and J. K. G. Watson, J. Mol. Spectrosc. 65, 65 (1977).
${ }^{71}$ M. Semczuk, X. Li, W. Gunton, M. Haw, N. S. Dattani, J. Witz, A. K. Mills, D. J. Jones, and K. W. Madison, Phys. Rev. A , 052505 (2013).

${ }^{72}$ A. Bernard, C. Effantin, J. d'Incan, G. Fabre, R. Stringat, and R. F. Barrow, Mol. Phys. 67, 1 (1989). 